\title{
Is myomectomy safe during cesarean section in large myomas?
}

\author{
Mehmet Baki Senturk ${ }^{1 *}$, Mehmet Sukru Budak², Yusuf Cakmak ${ }^{3}$, Kasım Turan $^{4}$
}

Abstract

Objectives: The rate of cesarean section, gravida and pregnancy age have been gradually increasing. Thus the rates of myoma have increased during cesarean. But it may difficult to make decision about myomectomy during cesarean especially large myomas because of complications. Therefore, several obstetricians do not recommend cesarean myomectomy, except in cases of small and pedunculated uterine fibroids that do not result in complications. The aim of this study to evaluate safety of myomectomy during emergency cesarean section in large myomas

Material and Methods: We examined retrospectively the results of 190 patients who underwent cesarean myomectomy in two non-tertiary hospitals in southeastern Anatolia. We compared complications, hemoglobin change, length of stay in hospital and blood transfusion according to diameter of myoma. Complication and blood transfusion rates in relation to the diameter of uterine fibroids were evaluated using Fisher's exact test. Changes in hemoglobin levels from baseline value and length of stay in hospital were compared using the Mann Whitney U test. Significance level was set at $\mathrm{P}<0.01$ and 0.05

Results: No significant association was found between complication rates and diameter of the fibroids $(\mathrm{P}=0.633)$. Similarly, no significant association was observed between the type of uterine fibroids and complication rates $(\mathrm{P}=1.000)$. We also found no significant difference among the changes in hemoglobin levels, length of stay in hospital and transfusion $(\mathrm{P}=0.835, \mathrm{p}=0.184, \mathrm{p}=0.633)$.

Conclusions: Myomectomy is safe procedure at the emergency cesarean section in large myoma with attention. Key Words: Cesarean section; Complications; Myoma

Keywords: Cesarean section; Complications; Myoma

\section{Introduction}

The incidence of uterine fibroids during pregnancy ranges from $1.6 \%$ to $10.7 \%(1-4)$. The rate of cesarean sections, pregnancy age and gravida has been gradually increasing, and thus, the chances of an obstetrician encountering uterine fibroids during cesarean section have increased (5). However, a negative stance towards performing cesarean myomectomy has traditionally existed due to the risk of persistent bleeding that may require hysterectomy and cause increased postoperative morbidity (6-8). Therefore, several surgeons (9-11) do not recommend cesarean myomectomy, except in cases of small and pedunculated uterine fibroids that do not result in excessive bleeding in the myoma bed.

Cesarean myomectomy in large myoma is also still considered to be high risk for massive hemorrhage, uterine atony and peripartum hysterectomy. On the other hand, many retrospective and prospective studies $(8,9,12-15)$ suggest that cesarean myomectomy can be performed safely and in a cost-effective manner in selected cases even in large myomas. In the southern region of Turkey, which has the highest population growth in the country, maternity hospitals predominantly treat obstetric patients.
In the present study, we reviewed the outcomes of cesarean myomectomy performed in patients complaining of pain who presented to the emergency gynecology department of two different centers. The safety of large cesarean myomectomy procedures was investigated

\section{Material and Methods}

This was retrospective study. We included pregnant women with myomas who delivered via emergency cesarean section at Batman and Diyarbakir Gynecology and Obstetrics Hospital between 2011 and 2014. All patients were initially evaluated in the emergency department. Diagnoses of uterine fibroid were established by ultrasonography performed prior to cesarean section or during surgery. Informed consent was obtained from all patients. Ages, gravid, parity, length of stay in hospital, gestational week, and reason for surgery indication were recorded for each patient. Laporotomy was made with Pfannenstiel incision. In all patients, the cesarean section was followed by myomectomy after closure of the lower uterine segment; hemostasis in the myoma bed was achieved by suturing. 
Myomectomies were performed using sharp dissection from the capsule with scissors. If major hemorrhage was observed during surgery, hemoglobin changes was assessed and blood transfusion was performed when required. Postoperative hemoglobin value was assessed 8 hours after surgery. Location, number, and diameter of uterine fibroids, as well as preoperative and postoperative hemoglobin values and development of complications, length of stay in hospital, blood transfusion rate were recorded. Complications were considered uterine atony and hemorrhage. Cases were assigned to one of two groups, according to the diameter of uterine fibroids $(<5$ or $\geqslant 5 \mathrm{~cm})(16)$. Development of complications, length of stay in hospital, transfusion and hemorrhage rates were evaluated in relation to the size and type of uterine fibroids. There was no need ethics committee approval because of retrospective design.

Statistical analyses were performed using the Number Cruncher Statistical System (NCSS) 2007 (Utah, USA). Complication and blood transfusion rates in relation to the diameter of uterine fibroids were evaluated using Fisher's exact test. Changes in hemoglobin levels from baseline value and differences between two groups, length of stay in hospital were compared between the two groups using the Mann Whitney U test, student $t$ test and Paired Samples test. Significance level was set at $\mathrm{P}<0.01$ and 0.05 .

Results

Total of number births were 18412 in Batman State Hospital, 58308 in Diyarbakir State Hospital while the number of cesarean section were 5255 and 15945 respectively. The cesaraen section ratios were $28.54 \%$ and $27.34 \%$ respectively. Both hospitals are non-tertiary center. The mean age of patients was 30.2. The numbers of previous pregnancy were differenced from 0 to 9 while the mean value was 2.27. The gestational age were differenced from 32 to 42. Patients' characters are presented in Table 1.

Most of reasons for cesarean section were previous cesarean section. The reasons for cesarean section were maternal, fetal and labor related factors. Maternal reasons were considered as placental ablation, previous cesarean section, patients' request, myomectomized patients, placenta previa and preeclapsia. Fetal reasons were considered fetal distress, mal-presentation, twin pregnancy, umbilical cord prolapsus and oligohydroamnios. Labor related factors were non-progressive labor and cephalopelvic disproportion (Table 2).

Among all patients, $154(81.1 \%)$ presented a solitary uterine fibroid, 31 (16.3\%) had two fibroids, 2 (1.0\%) had three fibroids, and $3(1.6 \%)$ had four fibroids. In addition, $12.6 \%$ (24) presented uterine fibroids with the largest one measuring $\geqslant 5 \mathrm{~cm}$, and $166(87.4 \%)$ showed uterine fibroids $<5 \mathrm{~cm}$. Of these patients, 86 $(45.3 \%)$ presented intramural fibroids, and 104 $(54.7 \%)$ had subserosal fibroids. Characteristics of uterine fibroids are presented in Table 3.
Among the 3 patients who developed hemorrhage, one presented an intramural fibroid measuring $7 \mathrm{~cm}$ in diameter, and 2 showed cornual fibroids measuring 4 and $5 \mathrm{~cm}$ in diameter, respectively. All of those patients required blood transfusion.

Eight patients who developed uterine atony also received blood transfusion. Ligation of the hypogastric artery was performed in only 3 patients who developed uterine atony; the rest of these patients were managed with uterine massage and oxytocin infusion. None of the patients required hysterectomy. Preoperative and postoperative hemoglobin changes are presented in table 4.

No significant association was found between complication rates and diameter of the fibroids $(\mathrm{P}=0.633) \quad$ (Table 5). Similarly, no significant association was observed between the type of uterine fibroids and complication rates $(\mathrm{P}=1.000)$ (Table 5) or between the diameter of uterine fibroids and the mean changes in hemoglobin levels from baseline value $(\mathrm{P}=0.835)$ (Table 6). There were also no significant differences between two groups in terms of blood transfusion rate and length of stay in hospital $(p=0.633$ and $\mathrm{p}=0.183$ respectively) (Table 6 ).

Table 1. Patients' characteristics

\begin{tabular}{lcc} 
& Min-Max & Mean \pm SD \\
Age (years) & $13-52$ & $30.92 \pm 6.1$ \\
Gravidity & $1-10$ & 5 \\
Parity & $0-9$ & $2.30 \pm 1.83$ \\
Gestational week & $32-42$ & $37.87 \pm 2.0$ \\
Length of Hospital & $1-6$ & 7 \\
Stay (days) & & $2.14 \pm 0.66$ \\
\hline
\end{tabular}

Table 2. Reason for cesarean section indication

$\begin{array}{lcc}\text { Fetal Reason } & \mathrm{n} & \% \\ \text { Maternal Reasons } & 46 & 24,4 \\ \text { The reasons about } & 126 & 66,2 \\ \text { labor } & 18 & 09,4\end{array}$

Table 3. Characteristics of uterine fibroids

\begin{tabular}{llc} 
& & \\
Type of Uterine & Intramural & $86(45,3)$ \\
Fibroids & Subserosal & $104(54,7)$ \\
Diameter of Uterine & $<5 \mathrm{~cm}$ & $166(87,4)$ \\
Fibroids & $\geq 5 \mathrm{~cm}$ & $24(12,6)$ \\
& Anterofundal & $6(3,2)$ \\
& Anteroisthmic & $2(1,1)$ \\
& Anterior & $57(30)$ \\
Location of Uterine & Fundal & $64(33,7)$ \\
Fibroids & Isthmus & $14(7,4)$ \\
& Cornual & $5(2,6)$ \\
& Posterior & $40(21,1)$ \\
& Cervical & $2(1)$ \\
\hline
\end{tabular}


Table 4. Preoperative and postoperative Hemoglobin changes

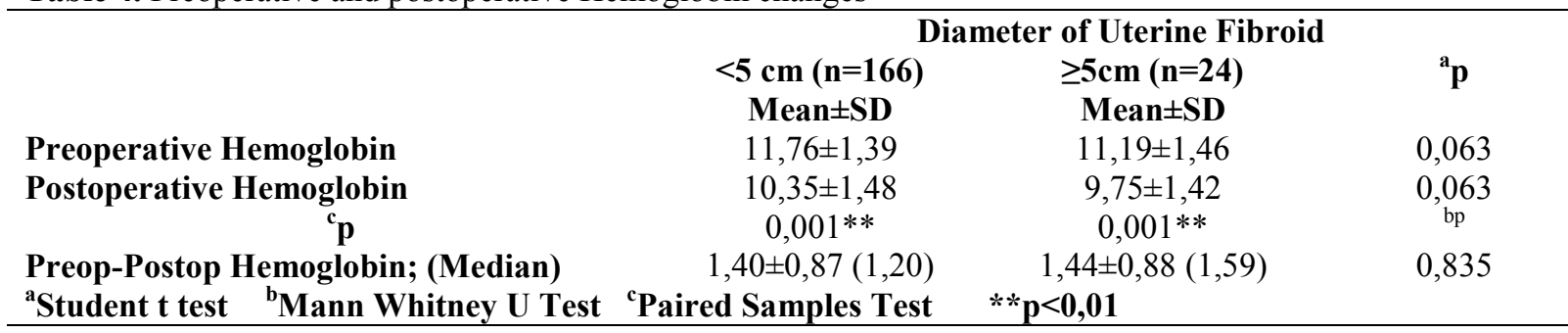

Table 5. Complications by type and diameters of uterine fibroids

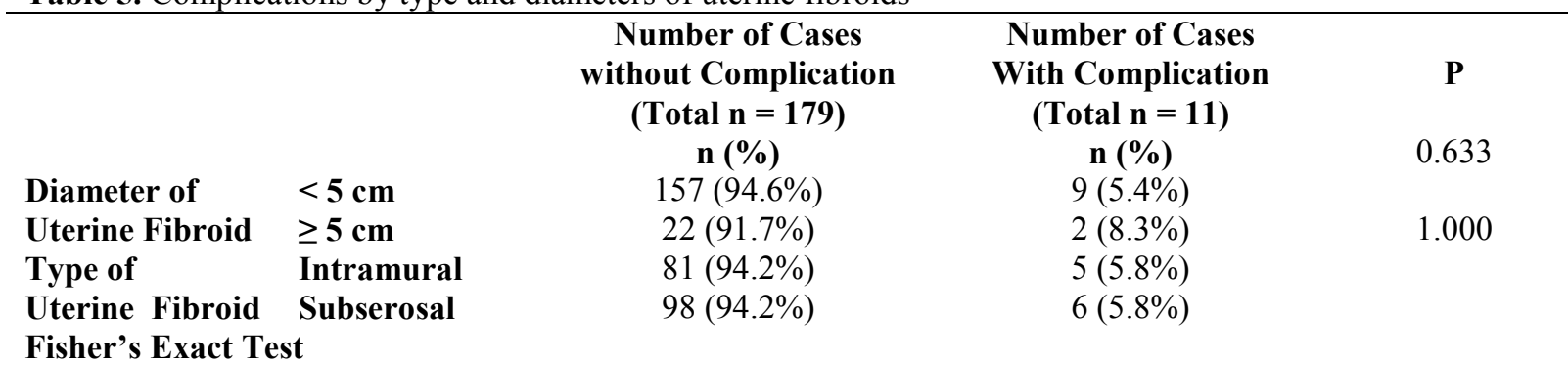

Table 6. Comparison of mean change in hemoglobin values from baseline value, length of stay in hospital and transfusion rate between patients with uterine fibroids $<5 \mathrm{~cm}$ and $\geq 5 \mathrm{~cm}$ in Diameter

\begin{tabular}{|c|c|c|c|c|}
\hline \multirow[b]{4}{*}{$\begin{array}{l}\text { Mean Change in Hemoglobin } \\
\text { baseline }\end{array}$} & \multirow[b]{4}{*}{ from } & \multicolumn{2}{|c|}{ Diameter of Uterine Fibroid } & \multirow[t]{3}{*}{$\mathrm{P}$} \\
\hline & & $<5 \mathrm{~cm}(\mathrm{n}=166)$ & $\geq 5 \mathrm{~cm}(\mathrm{n}=24)$ & \\
\hline & & Mean \pm SD (Median) & Mean \pm SD (Median) & \\
\hline & & $1.40 \pm 0.87(1.20)$ & $1.44 \pm 0.88(1.59)$ & ${ }^{\mathrm{a}} 0.835$ \\
\hline Length of stay in hospital (day) & & $2.37 \pm 2.34(2)$ & $2.25 \pm 0.44(2)$ & ${ }^{\mathrm{a}} 0.184$ \\
\hline Transfusion No & & $157(94.6)$ & $2.25 \pm 0.44(2)$ & ${ }^{b} 0.633$ \\
\hline Transfusion Yes & & $9(5.4)$ & $2(8.3)$ & \\
\hline
\end{tabular}

\section{Discussion}

This study demonstrated that there were no differences between large and small size myomectomy during cesarean section in terms of complications, changes in hemoglobin levels, length of stay in hospital and blood transfusion rate.

Most obstetricians are opposed to performing myomectomy during cesarean section, except for cases with pedunculated and small fibroids, owing to the risk of excessive hemorrhage that may require hysterectomy or result in mortality. On the other hand, if myomectomy is avoided, it may increase the patient's risk of another operation that may unfavorably affect future pregnancies (17). Especially, large myomas $>5 \mathrm{~cm}$ are associated with higher risk of adverse pregnancy complications such us preterm delivery, short cervix, premature rupture of membrane, postpartum bleeding when compared with small or no myomas (18). If safely performed, the procedure is cost-effective and can prevent morbidities associated with potential future surgeries. Myomectomy also reduces puerperal uterine involution and could decrease complications related to uterine fibroids, such as menorrhagia, anemia, and pain (19).
The safety of cesarean myomectomy has been previously studied. These studies have mostly been retrospective, with only a limited number of them being prospective in design. Cesarean myomectomy appears to be a safe procedure, as shown by retrospective studies involving small numbers of patients $(8,17,20,21)$. Similarly, in a study that evaluated 368 patients with uterine fibroids, 111 patients underwent myomectomy and 257 patients underwent cesarean section only patients were selected for myomectomy using similar criteria as in a previous study.

But this study (14) noted that myomectomy should be avoided in the presence of large fundal or intramural fibroids, and that cesarean myomectomy could be a safe procedure in selected cases in the hands of experienced surgeons. In both studies, myomectomy was performed at surgeons' discretion. In a meta-analysis of nine studies (11), cesarean myomectomy was an appropriate procedure for cases in which there were no risks of complication, as determined by experienced surgeons. Although that review (11) did not report cases of hysterectomy, it emphasized that the procedure would not have been an 
appropriate approach in patients with large intramural fibroids owing to the risk of massive hemorrhage and the consequent need of hysterectomy.

In another review (22), in which the majority of removed fibroids were pedunculated and had $<6 \mathrm{~cm}$ diameter, the authors note that great caution must be exercised while performing cesarean myomectomy, even if it appears to be a safe procedure, and they suggest that this procedure should be limited to cases of pedunculated or lower uterine segment fibroids that interfere with the evacuation of the fetus. There are some case reports on cesarean myomectomy in large myomas. Leanza et al. (23) demonstrated a successful cesarean myomectomy in a large myoma with $22 \mathrm{~cm}$ in diameter.

Ma et al. (24) also reported that cesarean myomectomy in a $40 \mathrm{~cm}$ sized moms was uneventful after the ligation of bilateral uterine arteries for the prevention bleeding. In a retrospective cohort study conducted by Kwon et al. (9) have shown that there were no statistical differences between cesarean myomectomy in large $(>5 \mathrm{~cm})$ and small $(<5 \mathrm{~cm})$ myomas in terms of hemoglobin changes, operative time, postoperative fever and hospitalized days.

Our study is meaningful, because we compared two groups to evaluate the safety of cesarean myomectomy in large myomas. Our study demonstrated that there were no differences between two groups in terms of complications, hemoglobin changes, blood transfusion and length of stay in hospital. Two patients with cornual fibroids and one patient with intramural fibroid $(7 \mathrm{~cm}$ in diameter) developed hemorrhage; this observation supports the concerns discussed in the literature. However, these patients did not require additional surgical procedures other than simple suturing but erythrocyte suspension was given to these patients.

Various approaches have been suggested to reduce the bleeding during cesarean myomectomy. In a study of 9 cases, (25) selective uterine artery de vascularization and ligation of ovari-proprium ligament appeared to reduce hemorrhage. Ligation of the ascending or descending uterine artery was performed depending on the location of uterine fibroids. None of the patients required hysterectomy, and only one patient received blood transfusion. In a controlled prospective study, (26) patients who had undergone cesarean myomectomy were compared to patients who had undergone uterine artery occlusion and, with the exception of operation times, no significant differences were reported between the two groups in terms of complications, such as hemorrhage rates and mean change in hemoglobin values from baseline. In that study, patients in the control group had undergone cesarean section only, and this fact was considered a study limitation. In a randomized study of 52 patients (27), bilateral ligations of the ascending uterine arteries were compared to tourniquet application. Although one patient in the tourniquet group required bilateral ligation of the hypogastric artery postoperatively, no significant difference was observed between the two groups in terms of hemorrhage or other complications. In our study, 8 patients developed uterine atony and 3 patients underwent bilateral ligation of the hypogastric artery. Bilateral ligation of the hypogastric arteries has been used to control uterine hemorrhage by reducing the pulse pressure of blood flowing to the uterus (28). The technique is challenging even for an experienced pelvic surgeon, especially when there is a large uterus, a transverse lower abdominal incision, ongoing pelvic hemorrhage, or the patient has a high body mass index. Successful and safe bilateral hypogastric ligation becomes even more difficult when attempted by a surgeon who rarely operates deep in the pelvic retroperitoneal space (29). For these reasons, uterine compression sutures and, less commonly, uterine artery ligation, have largely replaced this procedure as first-line surgical options.

Among the patients who developed hemorrhage, 3 presented cornual uterine fibroids and one had an intramural fibroid $(7 \mathrm{~cm}$ in diameter). This patients did not require additional surgical procedures other than the suturing of the myoma bed.

The most important limitation of the present study is its retrospective design. The most important advantage of the present study is that studied patients had no antenatal data and were diagnosed with uterine fibroids shortly after presentation to the emergency room or during surgery. This has raised the question of whether random cesarean myomectomy could be a safe procedure even in large myomas. Patients with placenta previa totalis and placental detachment also underwent myomectomy, suggesting that selection criteria were less stringent than those used in previous studies.

\section{Conclusion}

Our results suggest that myomectomy in large sized is a safe procedure under emergency conditions during cesarean section. However, large intramural or cornual fibroids must be approached with great caution. In such cases, bilateral ligation of the uterine artery or ligation of the hypogastric artery together with ligation of the ovarian ligament should be considered before proceeding with myomectomy in order to reduce hemorrhage. Finally, recommend performing myomectomy under conditions in which blood products are readily available

Acknowledgements: We want to thanks to archives officers of Batman and Diyarbakir states hospital for their help. This study was not supported by any company . The language has been revised by natural English speaker Caroline Jane Walker. Statistical Analysis has been perform by Empiar Statistical office (Email: info@empiaristatistik.com)

Conflict of Interest: The authors declare no conflicts of interest. 


\section{References}

1. Exacoustòs C, Rosati P. Ultrasound diagnosis of uterine myomas and complications in pregnancy. Obstet Gynecol. 1993;82(1):97-101

2. Strobelt N, Ghidini A, Cavallone M, .Pensabene I, ceruti P, Vergani P. Natural history of uterine leiomyomas in pregnancy. J Ultrasound Med. 1994;13(5):399-401.

3. Qidwai GI, Caughey AB, Jacoby AF. Obstetric outcomes in women with sonographically identified uterine leiomyomata. Obstet Gynecol. 2006;107(2 Pt 1):376-82.

4. Laughlin SK, Baird DD, Savitz DA, Herring AH, Hartmann KE. Prevalence of uterine leiomyomas in the first trimester of pregnancy: an ultrasound-screening study. Obstet Gynecol. 2009;113(3):630-5

5. Coleman-Cowger VH, Erickson K, Spong CY, Portnoy B Croswell J, Schulkin J. Current practice of cesarean delivery on maternal request following the 2006 state-ofthe science conference. J Reprod Med. 2010;55(1-2):25-30.

6. Sheiner E, Bashiri A, Levy A, Hershkovitz R, Katz M, Mazor M. Obstetric characteristics and perinatal outcome of pregnancies with uterine leiomyomas. J Reprod Med 2004;49:182-6.

7. Davis JL, Ray-Mazumder S, Hobel CJ, Baley K, Sassoon D. Uterine leiomyomas in pregnancy: a prospective study. Obstet Gynecol 1990;75:41-4.

8. Ortac F, Gungor M, Sonmezer M. Myomectomy during cesarean section. Int J Gynaecol Obstet 1999;67:189-90.

9. Kwon DH, Song JE, Yoon KR, Lee KY. The safety of cesarean myomectomy in women with large myomas. Obstet Gynecol Sci. 2014;57(5):367-72.

10. Kaymak O, Ustunyurt E, Okyay RE, kalyoncu S, Mollamahmutoğku L. Myomectomy during cesarean section. Int J Gynaecol Obstet. 2005;89(2):90-3.

11. Song D, Zhang W, Chames MC, Gou J. Myomectomy during cesarean delivery. Int $\mathrm{J}$ Gynaecol Obstet. 2013;121(3):208-13

12. Burton CA, Grimes DA, March CM. Surgical management of leiomyomata during pregnancy. Obstet Gynecol. 1989;74(5):707-9

13. Febo G, Tessarolo M, Leo L, Arduino S, Wierdis T, Lanza L. Surgical management of leiomyomata in pregnancy. Clin Exp Obstet Gynecol 1997;24(2):76-8.

14. Roman AS, Tabsh KM. Myomectomy at time of cesarean delivery: a retrospective cohort study.BMC Pregnancy Childbirth. 2004;16;4(1):14

15. Hassiakos D, Christopoulos P, Vitoratos N, Xarchoulakou E, Vaggos G, Papadias K. Myomectomy during cesarean section: a safe procedure? Ann N Y Acad Sci.2006;1092:408-13.
16. Vergani P, Locatelli A, Ghidini A, Andreani M, Sala F, Pezzullo JC. Large uterine leiomyomata and risk of cesarean delivery. Obstet Gynecol 2007;109(2 Pt 1):410-4

17. Park BJ, Kim YW. Safety of cesarean myomectomy. J Obstet Gynaecol Res. 2009;35(5):906-11

18. Shavell VI, Thakur M, Sawant A, Kruger ML, Jones TB, Singh M et al.. Adverse obstetric outcomes associated with sonographically identified large uterine fibroids. Fertil Steril 2012;97:107-10.

19. Kwawukume EY. Caesarean myomectomy. Afr J Reprod Health. 2002;6(3):38-43.

20. Celal K1, Hülya C. The evaluation of myomectomies performed during cesarean section in our clinic. Niger Med J. 2011;52(3):186-8

21. Incebiyik A, Hilali NG, Camuzcuoglu A, Vural M, Camuzcuoglu H. Myomectomy during caesarean: a retrospective evaluation of 16 cases. Arch Gynecol Obstet. 2014;289(3):569-73

22. Awoleke JO. Myomectomy during Caesarean Birth in Fibroid-Endemic, Low-Resource Settings. Obstet Gynecol Int. 2013:520834. doi: 10.1155/2013/520834

23. Leanza V, Fichera S, Leanza G, Cannizzaro MA. Huge fibroid (g. 3.000) removed during cesarean section with uterus preservation: a case report. Ann Ital Chir $2011 ; 82: 75-7$

24. Ma PC, Juan YC, Wang ID, Chen CH, Liu WM, Jeng CJ. A huge leiomyoma subjected to a myomectomy during a cesarean section. Taiwan J Obstet Gynecol 2010;49:220-2.

25. Desai BR, Patted SS, Pujar YV, Sherigar BY, Das SR, Ruge JC. A novel technique of selective uterine devascularization before myomectomy at the time of cesarean section: a pilot study. Fertil Steril. 2010;94(1):362-4

26. Lin JY, Lee WL, Wang PH, Lai MJ, Chang WH, Liu WM. Uterine artery occlusion and myomectomy for treatment of pregnant women with uterine leiomyomas who are undergoing cesarean section. J Obstet Gynaecol Res. 2010;36(2):284-90

27. Sapmaz E, Celik H, Altungül A. Bilateral ascending uterine artery ligation vs. tourniquet use for hemostasis in cesarean myomectomy. A comparison.J Reprod Med. 2003;48(12):950-4.

28. Evans S, McShane P. The efficacy of internal iliac artery ligation in obstetric hemorrhage . Surg Gynecol Obstet $1985 ; 160: 250$

29. Joshi VM, Otiv SR, Majumder R, Nikam YA, Shrivastana $\mathrm{M}$. İnternal iliac artery ligation for arresting postpartum hemorrhage. BJOG 2007; 114:356

Copyright (C) 2014 The Author(s); This is an open-access article distributed under the terms of the Creative Commons Attribution License (http://creativecommons.org/licenses/by/4.0), which permits unrestricted use, distribution, and reproduction in any medium, provided the original work is properly cited. All Rights reserved by international journal of Medical Science and Discovery and Lycians Press Inc. 